\title{
Militarismus, Nietzsche und die soziale Revolution. Über die Deutschlandbilder in den ersten Jahrgängen der Zeitschrift Neue Jugend
}

\section{Einführung}

Im Jahre 1915 gründete Chen Duxiu, der kurz zuvor aus dem Exil in Japan zurückgekehrt war, in Shanghai eine neue Zeitschrift, die seitdem die kulturelle und gesellschaftliche Entwicklung Chinas maßgeblich geprägt hat. Sie wird oft im chinesischen Volksmund einstimmig als die „Zeitschrift aller Zeitschriften“ gepriesen. Trotz des Titelwechsels von Zeitschrift für die Jugend zu Neuer Jugend ab dem zweiten Jahrgang bleibt ihr fremdsprachiger Name „La Jeunesse“ (im Folgenden als Neue Jugend bezeichnet) bis in die 4.-Mai-Bewegung unverändert (Abb. 1 und 2). Schon für das allererste Heft dieser Zeitschrift verfasste Chen einen Aufsatz über „Die Franzosen und die moderne Zivilisation“, in dem er die These vertritt, dass die drei Hauptlehren der modernen Zivilisation, nämlich die Menschenrechte, die Evolutionstheorie sowie der Sozialismus, allesamt den Franzosen zu verdanken seien (vgl. Chen 1915b). Außerdem übersetzt Chen Auszüge aus Charles Seignobos' Histoire de la Civilisation Contemporaine, die er ebenfalls im Eröffnungsheft der Neuen Jugend publiziert. Da viele von Chens Mitstreitern in der Neue-Kultur-Bewegung in Frankreich studiert haben, resümiert der renommierte Historiker Tse-tsung Chow:

Chinese intellectuals in the May Fourth Movement were, in many cases, overwhelmingly dominated by the democratic ideas and liberalism of eighteenth- and nineteenth-century France rather than the ideas of other Western countries. The temper of the Chinese intellectuals in the movement often bore traces of French romanticism.

(Chow 1960, 36)

Insofern scheint es wichtig, die Vorstellung und Rezeption von Frankreich und dessen intellektuellen Strömungen während der 4.-Mai-Bewegung zu untersuchen.

Allerdings ist es auffällig, dass das Interesse zumindest der Redaktion der Neuen Jugend für Deutschland größer ist als das für Frankreich. Bereits im ersten Heft wird eine Rubrik mit dem Titel „Aus aller Welt“ eröffnet, in der eine Reihe von kleinen landeskundlichen Texten erscheint. An erster Stelle wird hier nicht Frankreich, sondern Deutschland dem chinesischen Lesepublikum vorgestellt. Diese von Li Yimin zusammengestellten Texte, neun an der Zahl, 
befassen sich mit Kaiser Wilhelm II., dem Berliner Schloss, der Stadt Berlin, den Verkehrsmitteln, den Parkanlagen, dem Silvester in Berlin, dem Duellverbot sowie dem deutschen Militärwesen (Li 1915a). Im zweiten Heft wird diese Reihe fortgesetzt und um zwei weitere Texte jeweils über die sozialdemokratische Partei und die Siegessäule ergänzt (Li 1915b). Deshalb ist es naheliegend, sich mit dem Deutschlandbild der Neuen Jugend auseinanderzusetzen.

In der bisherigen Forschung wird vor allem durch die beiden bedeutenden Sinologen Mechthild Leutner und Andreas Steen auf die historische Überlieferung eines emotional meist mit Verehrung aufgeladenen Deutschlandbilds der chinesischen Intellektuellen am Anfang des 20. Jahrhunderts aufmerksam gemacht. Sie weisen darauf hin, dass das chinesische Deutschlandbild „lange Zeit von einer Anerkennung und Bewunderung Deutschlands geprägt“ sei: „Militärische Leistungen, die Einigung Deutschlands, der Aufstieg Preußens und das herrschende autokratische System der konstitutionellen Monarchie galten vielen Intellektuellen als Vorbild für den zukünftigen Weg Chinas“ (Leutner und Steen 2006, 496).

Der Berliner Sinologe Roland Felber hat das Deutschlandbild vor und nach der 4.-Mai-Bewegung untersucht und die Unterschiede in den konservativen und revolutionären Interpretationen des deutschen Militarismus herausgearbeitet (Felber 1999). Darüber hinaus hat der Shanghaier Geschichtswissenschaftler Zhao Bing den Wandel des „deutschen Geistes“ während der Neue-Kultur-Bewegung insgesamt umrissen - ohne jedoch diesen berühmt-berüchtigten Geist näher zu bestimmen (Zhao 2017). Die Aufgabe der vorliegenden Untersuchung wird also sein, diesen Wandel an einem konkreten Beispiel - nämlich den ersten sechs Jahrgängen der Neuen Jugend von September 1915 bis Mai 1919 - darzustellen. Daran wird deutlich, wie sich die chinesischen Intellektuellen vor hundert Jahren das Gedankengut Deutschlands angeeignet haben, um daraus einen gangbaren Weg zur Erneuerung und Umgestaltung Chinas zu finden.

\section{Das Interesse der Neuen Jugend am deutschen Militarismus}

Es gehört zum Konsens der Forschung, dass die chinesischen Intellektuellen im frühen 20. Jahrhundert gleichsam vom deutschen Militarismus geschwärmt haben (vgl. Kirby 1984). Schon in den 1870er Jahren zogen die von Wang Tao herausgegebenen Berichte des Preußisch-Französischen Kriegs viel Aufmerksamkeit sowohl der Politik als auch der akademischen Welt auf sich. Auch haben Vertreter der Yang-Wu-Bewegung, etwa Zhang Zhidong oder Yuan Shikai, ihre Truppen mit deutschen Waffen ausgerüstet und nach deutschem Muster trainiert. 


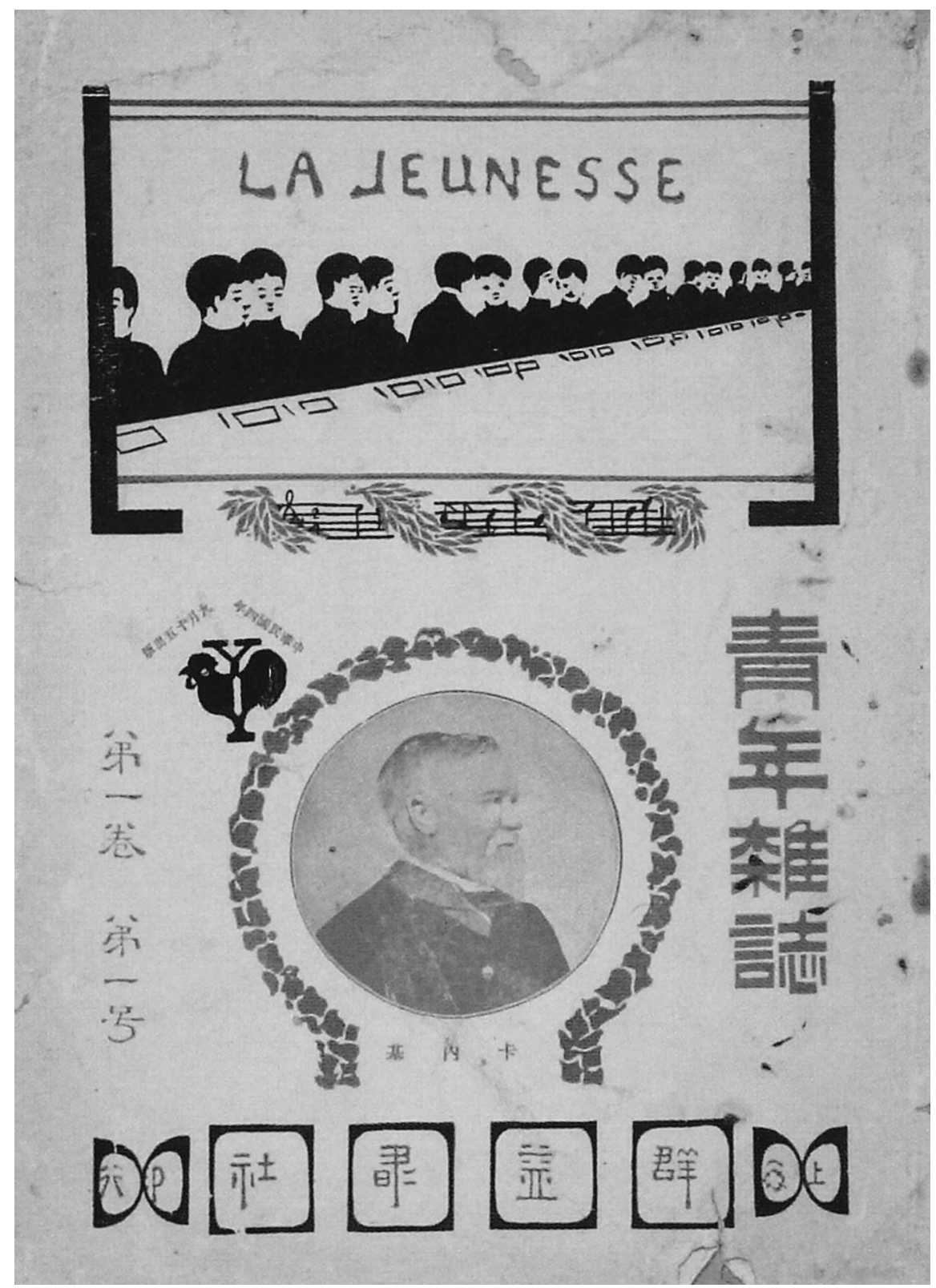

Abb. 1: Umschlag des Debüthefts der Zeitschrift für die Jugend (September 1915). 


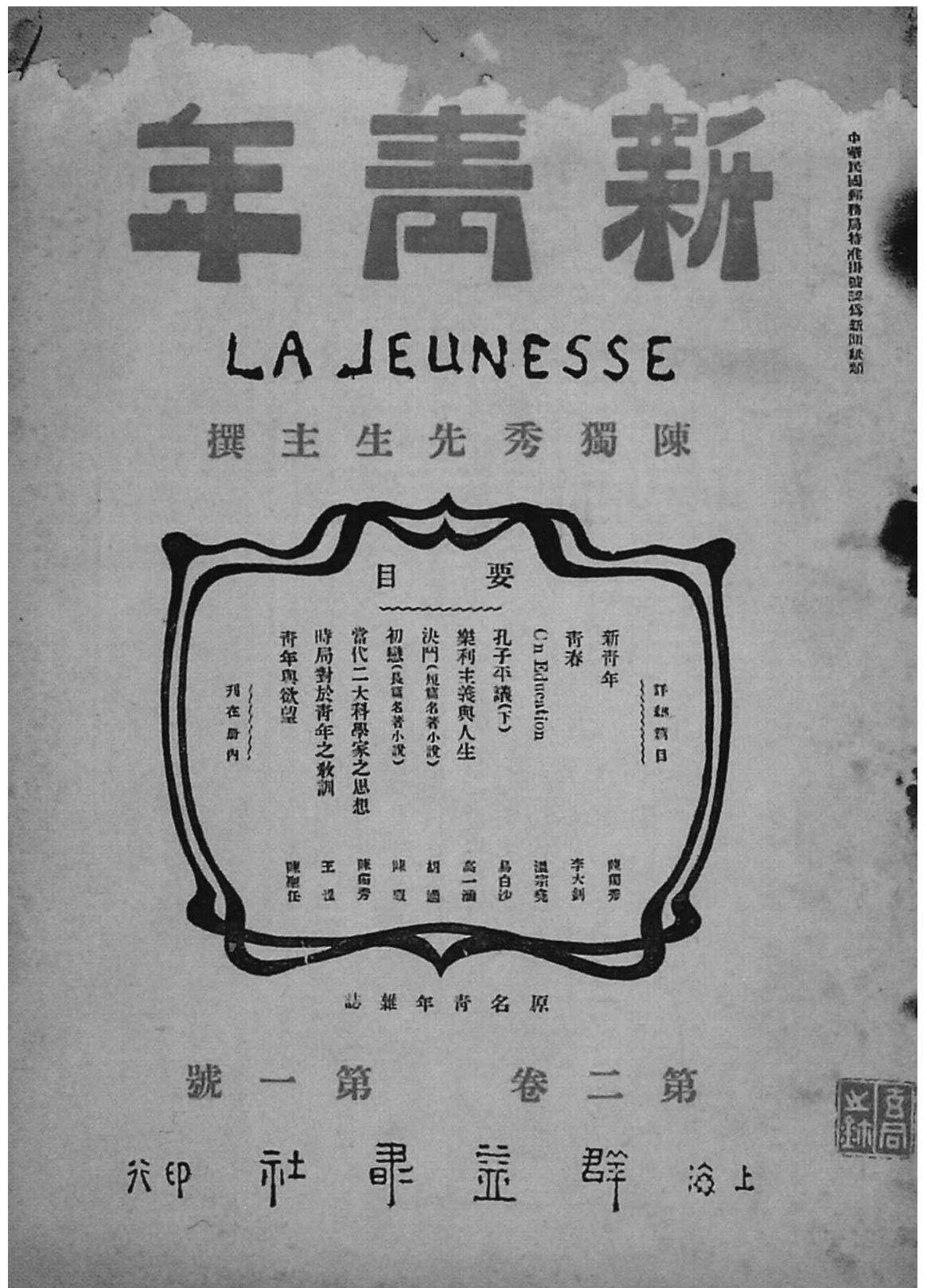

Abb. 2: Umschlag des 1. Hefts des 2. Jahrgangs der nun umbenannten Zeitschrift Neue Jugend (September 1916). 
Diese Vorbildfunktion des deutschen Militärs ist auf den unaufhaltsamen Aufstieg Preußens und die Vereinigung Deutschlands unter dessen Leitung zurückzuführen. Das hat man in China, das damals noch nach einem Weg zur Erneuerung und Selbststärkung suchte, als große Ermutigung der eigenen Bestrebungen empfunden. Charakteristisch hierfür ist jene Ansicht, die Liang Qichao in seinem Büchlein Der neue Bürger vertritt: Als ein „neues Land“ sei Deutschland nur deshalb imstande, „Österreich zu zerschlagen und Frankreich zu unterwerfen und so erhaben über Europa zu schauen“, weil Bismarck seine Politik von „Eisen und Blut“ mit Konsequenz durchgesetzt habe (Liang 1994, 147). ${ }^{1}$ Die Forderung, dass China Deutschlands Beispiel folgen sollte, wird hier zwar nicht ausgesprochen, lässt sich aber schon deutlich vernehmen.

Folgerichtig wundert es nicht, dass Li Yimin in der Rubrik „Aus aller Welt“ zunächst die allgemeine Soldatenverehrung der deutschen Gesellschaft hervorhebt. Im Abschnitt „Die deutschen Soldaten“ resümiert Li:

Für die Deutschen sind die Soldaten wie die Gebildeten bei uns. Wir Chinesen halten seit jeher die Gebildeten für die Spitze aller vier Stände. Ihnen wird von den obersten bis zu den untersten Schichten mit Bewunderung begegnet. [. . . ] Ungefähr genauso werden die Soldaten in Deutschland verehrt.

(Li 1915a, 70)

Diese Darstellung ist offenbar keine Übertreibung, sondern spiegelt wohl die Realität der deutschen Gesellschaft vor dem Ersten Weltkrieg ziemlich genau wider. Im Zeitalter der Reichsgründung sei, wie Friedrich Meinecke in seiner bedeutenden Schrift Die deutsche Katastrophe beschreibt, eine Weihe auf dem preußischen Militarismus gelegen, und „der preußische Leutnant ging als junger Gott, der bürgerliche Reserveleutnant wenigstens als Halbgott durch die Welt“ (Meinecke 1969, 336). Li Yimin zufolge ist gerade diese Vergötterung der Offiziere der Grund für die siegreichen Feldzüge des Kaiserreichs am Anfang des Ersten Weltkrieges:

Die deutsche Gesellschaft ist eine utilitaristische, eine militärische Gesellschaft. [. . .] Als der Europäische Krieg ausbrach, konnten viele nicht glauben, dass das deutsche Heer den erbitterten Kampf überstehen würde. Die Erfahrung von heute ist genau das Gegenteil. Der Grund liegt darin, dass das Militär sich selbst schätzt, da es geschätzt wird. Alle Herzen schließen sich fest zusammen und dadurch entsteht das, was zum Triumph führt.

(Li 1915a, 70)

Im zweiten Heft des ersten Jahrgangs stellt Li dem chinesischen Lesepublikum die Siegessäule vor, im vierten Heft veröffentlicht Pan Zanhua unter dem Pseudonym

1 Chinesischsprachige Titel und Zitate in diesem Beitrag wurden vom Verfasser selbst ins Deutsche übersetzt. 
Pan Zan einen Aufsatz mit dem Titel „Der deutsche Militärgott Hindenburg“, und im sechsten Heft publizierte Li dann schließlich einen weiteren Artikel über August von Mackensen. Der lobende, hochgestimmte Ton der letzten beiden Texte ist bezeichnend. So beschreibt Pan etwa Hindenburg als „Krieger aller Krieger, Schutzgott der Nation, von der Welt als Essenz des preußischen Geists gepriesen“ (Pan 1915, 282). Detailliert werden Hindenburgs Befehle und taktische Entscheidungen während der Schlacht bei Tannenberg dokumentiert. Daraus zieht Pan die Schlussfolgerung: „Die Deutschen ehren General von Hindenburg weit mehr als den Kaiser“ (Pan 1915, 284). Ein weiteres Zeichen für die Idealisierung der Befehlshaber der kaiserlichen Armee ist der Sachverhalt, dass beide Aufsätze mit Abbildungen ausgestattet sind (Abb. 3 und 4). Das ganzseitige Porträt von Hindenburg zeigt ihn nach der Schlacht von Tannenberg bei der Planung weiterer Manöver und ist mit seinem Zitat versehen: „Möge uns der Geist von 1914/15 erhalten bleiben!“ Dass ein Porträt eine ganze Seite in Anspruch nimmt, ist in den ersten Jahrgängen der Neuen Jugend äußerst selten.

Nicht nur den Persönlichkeiten, sondern auch den anfänglichen Siegeszügen des wilhelminischen Kaiserreichs galt die Faszination chinesischer Intellektueller angesichts seines Militärwesens. Trotz seines Vorbehalts gegenüber dem Militarismus zeigt sich Chen Duxiu Anfang 1916 zuversichtlich, dass das Kaiserreich den Ersten Weltkrieg gewinnen werde:

Der Europäische Krieg hat sich auf die ganze Welt ausgeweitet und das Los von Sieg und Niederlage wird immer deutlicher. Die Deutschen haben außer Tsingtau sowie Kolonien in Südafrika und Ozeanien keine einzige Landfläche verloren. Sie haben an der Westfront die Briten und Franzosen fern von der eigenen Grenze gehalten und sind im Osten tausend Meilen auf russischen Boden vorgedrungen. Sie zogen in den Balkan ein und haben Serbien vernichtet. Die Hauptstädte von Deutschland und der Türkei sind mit Eisenbahnen verbunden. Obwohl die Elitetruppen nach Süden gerückt sind, vermögen Russland im Osten, England und Frankreich im Westen nur ihre letzten Atemzüge zu tun und sonst nichts auszurichten.

(Chen 1916, 305-306)

Kaum vorstellbar, dass der Herausgeber einer Zeitschrift mit französischem Namen die Feldzüge Deutschlands derart hochstilisiert. Aber diese feierlichen Töne werden noch einmal überboten durch den Beitrag von Liu Shuya im 3. Heft des 2. Jahrgangs mit dem Titel „Militarismus“:

Seit dem Kriegsbeginn hat Deutschland Belgien vernichtet, Frankreich unterjocht und Russland in die Knie gezwungen. Umringt von Feinden musste es sich aller Streitkräfte der Welt erwehren. Dennoch ist es Deutschland gelungen, seine Feinde zu zerschlagen und deren Land zu erobern. [. . .] Solche großen Siege hat es in der Tat seit Beginn der Geschichtsschreibung noch nie gegeben.

(Liu 1916b, 166) 
Allerdings interessieren sich die Beitragenden der Neuen Jugend weniger für die technische Seite der Kriegsführung als vielmehr für die kriegerische Mentalität. Der hier konstruierte Mythos von der Unschlagbarkeit des deutschen Militärs dient nicht dazu, das chinesische Heerwesen zu erneuern, sondern die Jugend. Vor allem sollen den jungen Chinesen Opferbereitschaft und Kampfgeist beigebracht werden. Gerade in diesem Sinne stellte Xie Hong (1915) im dritten Heft des ersten Bandes den „Jungdeutschland-Bund“ vor, eine von Colmar von der Goltz Pascha ins Leben gerufene Organisation, die bis 1914 mehr als 750.000 Mitglieder zählte. Auf diese bemerkenswerte zeitliche Parallelität der Jugendbewegung Chinas und Deutschlands wurde in der Forschung bereits hingewiesen (vgl. Cao 2013). Für Xie ist das Ausschlaggebende nicht das militärische Training, das die Jugendlichen auf den bevorstehenden Kriegseinsatz vorbereitet, sondern die Bildung einer „deutschen Seele“, aufgrund derer das ganze Volk vor keiner Auseinandersetzung zurückscheue und bereit sei, ,alles, was es an Geist, Fähigkeit und Vermögen besitzt, dem Staat zu opfern“ (Xie 1915, 193).

Diese Zielsetzung entspricht gänzlich der Ansicht Chen Duxius, der mit Nachdruck auf den Unterschied zwischen der westlichen und der östlichen Mentalität hinweist: Gerade weil die Deutschen „ihre heutigen Glorien durch Blut erworben“ hätten, sollte die chinesische Jugend ihrem Beispiel folgen, die ostasiatische Mentalität nach dem Motto „den Kampf scheuend lieber die Demütigung dulden“ ablegen und dem westlichen Charakter nacheifern, sprich „die Duldung verachten und lieber im Kampf fallen“ (Chen 1915c, 231). Noch unverhohlener formuliert Liu Shuya in seinem Aufsatz „Europäischer Krieg und das Bewusstsein der Jugend":

Kriegslust ist eine Tugend, Friedensliebe ein Verbrechen. Unter den Europäern sind die Deutschen am kriegslustigsten, daher sind sie auch am mächtigsten. [. . . ] Unter allen Völkern der Welt lieben wir - die Chinesen - den Frieden am ehesten. Daher sind wir auch am schwächsten. Wenn wir nicht dringend aus dieser Halluzination erwachen, können wir nie mehr der Zerstörung unserer Nation und der Auslöschung unserer Stämme entrinnen.

(Liu 1916a, 94)

Folgerichtig fordert Liu Shuya, mit Hilfe des deutschen Militarismus die Jugend Chinas „zum kriegslustigsten Volk der Welt“ umzumodeln. Diese Forderung stimmt mit Chen Duxius Mahnung an die Jugend nach seinem Rückblick auf die anfänglich unaufhaltbaren Feldzüge des Kaiserreichs überein: Die Jugend solle „die Stellung des Eroberers anstatt der des Eroberten“ (Chen 1916, 306) einnehmen. 


\section{像省 師 元 堡 登興 國德}

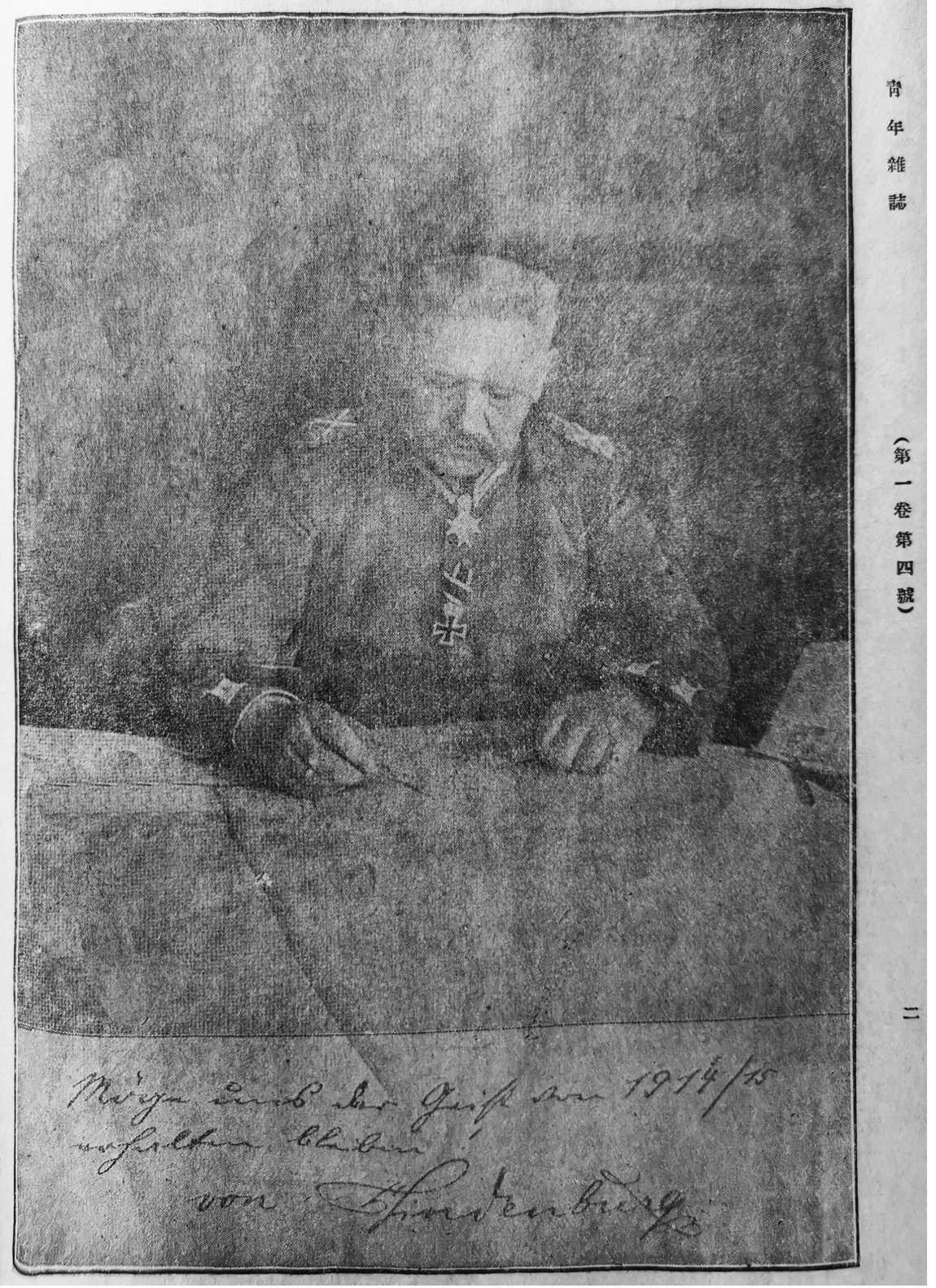

Abb. 3: Das Hindenburg-Porträt im 4. Heft des 1. Jahrgangs der Zeitschrift für die Jugend. 


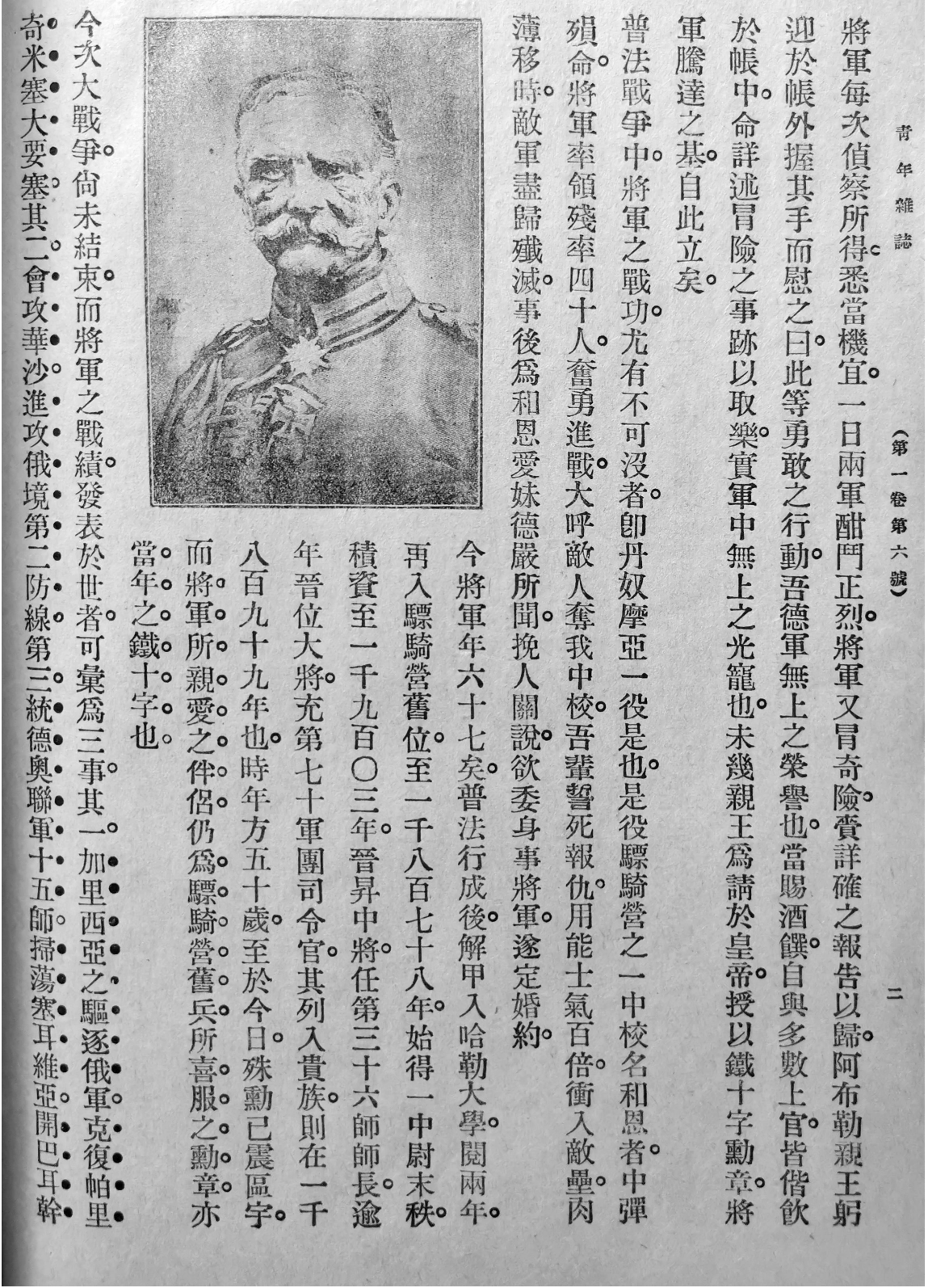

Abb. 4: Das Mackensen-Porträt im 6. Heft des ersten Jahrgangs der Zeitschrift für die Jugend. 


\section{Nietzsche}

Die Aufgabe, die Jugend nach dem Vorbild des deutschen Militarismus zu regenerieren, hängt des Weiteren mit der Befreiungsintention der Neuen Jugend zusammen. Der Aufruf zum Kampf gilt nicht einer gegnerischen Nation, sondern der eigenen Kulturtradition, deren überlieferter Tugendkanon als Einschränkung und Unterjochung empfunden wird. Die kriegerische Mentalität sollte der neuen Jugend zu einem absoluten Bruch mit den überlieferten ethischen Forderungen verhelfen, die als veraltet, ja knechtisch galten und deshalb von der jungen Generation abgelehnt wurden. Dabei wird der Militarismus von der Einführung der Philosophie Friedrich Nietzsches flankiert. In der Ankündigung des Herausgebers mit dem Titel „Aufruf an die Jugend“ stellt Chen Duxiu insgesamt sechs Lehren für die Jugend auf, deren allererste ein Aufruf zur Selbstemanzipation ist: Die Jugend solle sich „zu einem selbstständigen und keinesfalls mehr sklavischen Subjekt“" entwickeln (Chen 1915a, 2). Chen argumentiert mit Nietzsches Kategorien der Herren- und Sklaven-Moral: Er beurteilt die traditionellen chinesischen Wertnormen sowie Wunschvorstellungen wie etwa die unbedingte Loyalität oder ein gemildertes Straf- und Steuersystem unter der Herrschaft eines gnädigen Souveräns abwertend als „Sklavenmoral“ und „Sklavenglück“, weil diese Moral lediglich dazu diene, das Leiden des irdischen Daseins erträglich zu machen. In diesem Kontext geht es Chen weniger um die Kritik des christlichen Moralgefüges als vielmehr um die Emanzipation des Individuums aus der Enge der fremdbestimmten, festgelegten Normen. In einem weiteren Aufsatz, „Über den wahren Sinn des Lebens“, bringt Chen sein dezidiertes Verlangen nach Individualität noch einmal zum Ausdruck:

Oder wie jener Deutsche namens Nietzsche, der ebenfalls fordert, den individuellen Willen zu respektieren, das individuelle Talent zu entfalten, um sich zu einem großen Künstler, einem großen Unternehmer zu entwickeln - in einem Wort, zu einem dem gemeinen Volk übergeordneten Übermenschen zu werden - und dadurch den Sinn des Lebens zu erfüllen. Alle Phrasen von Gerechtigkeit und Moral sind nichts anderes als Lügen.

(Chen 1918a, 71)

Nach dem Verständnis Chens und der mit ihm revolutionär gesinnten Generation müssen auch in China die regulierenden sozialen Normen zugunsten einer umfassenden Entfaltung der eigenen Subjektivität außer Kraft gesetzt werden. Dieser programmatische Subjektivismus geht einher mit der Begeisterung für den deutschen Militarismus. Beim Kampf gegen die eigene Kulturtradition steht der jungen chinesischen Generation dann wieder die Philosophie Nietzsches als Waffe zur Verfügung. Schon seit Beginn des 20. Jahrhunderts wurde Nietzsche von namhaften Intellektuellen wie Wang Guowei, Liang Qichao oder Lu 
Xun dem chinesischen Lesepublikum vorgestellt. Insbesondere wurden die Zerstörung aller Idole und die Aufhebung aller normativen Setzungen als Kernthese Nietzsches aufgenommen und weiterverbreitet. Die drei Verwandlungen des Geistes, wie Nietzsche sie in seinem Also sprach Zarathustra schildert, aber vor allem die Gegenüberstellung von dem Löwen des „Ich will“ und dem Drachen des „Du sollst“ stehen im Einklang mit der traditionsskeptischen, ja überlieferungsfeindlichen Haltung der Neuen Jugend. Die große chinesische Komparatistin Yue Daiyu (1980) hat mit Recht resümiert, dass Nietzsche in seiner Interpretation durch die Vertreter der 4.-Mai-Bewegung die Gestalt eines triumphierenden Idolzerstörers annimmt, weil die Forderung nach einer „Umwertung aller Werte“ für die chinesischen Intellektuellen, die mit den kulturellen und ethischen Überlieferungen abzurechnen gedachten, einen Befreiungsschlag bedeute. Der slowakische Sinologe Marián Gálik (2017) hat in seinen Ausführungen diese Beobachtung bestätigt. Die gedankliche Nähe zwischen Nietzsches „heiligem Nein“ und den Kampfparolen während der Neue-Kultur-Bewegung - etwa „Reißt den Laden des Konfuzianismus nieder!“ - ist derart evident, dass diese traditionskritische Haltung zum Grundgestus einer gesamten Bewegung wurde. In der ersten Ausgabe der Neuen Jugend nach der 4.-Mai-Bewegung veröffentlicht $\mathrm{Hu}$ Shi einen Aufsatz über „Die Bedeutung der neuen Denkströmung“ und fasst dieses neue Denken als Gestus einer umfassenden Kritik zusammen:

Nietzsche sagte, das heutige Zeitalter sei eines der Umwertung aller Werte. Die Umwertung aller Werte ist die beste Deutung für die kritische Haltung. [. . .] Ich bin der Auffassung, dass die neuen Strömungen, wie unterschiedlich sie auch sein mögen, doch in einem Punkt miteinander übereinstimmen: nämlich in der kritischen Haltung.

(Hu 1919, 4)

\section{Sozialdarwinismus}

Die Bewunderung des deutschen Militarismus und der Philosophie Nietzsches durch die jungen chinesischen Intellektuellen steht allerdings deutlich unter dem Zeichen des Sozialdarwinismus, der seit der Übersetzung von Huxleys und Herbert Spencers Schriften im China der Jahrhundertwende recht schnell populär geworden ist (Wang 2010). Neben diesen beiden einflussreichen Autoren vertritt auch Ernst Haeckel, dessen Lehre von der Phylogenie unter anderem von Lu Xun (2005) rezipiert wurde, die Anwendung der Evolutionstheorie auf die Entwicklungsgeschichte menschlicher Gesellschaften. Bekanntlich hat sich der Sozialdarwinismus das Motto des „Survival of the Fittest“ auf die Fahnen geschrieben und das Theorem der natürlichen Selektion auf die zwischen- 
menschliche Ebene übertragen. Unter diesem Gesichtspunkt wären die internationalen Beziehungen als vollkommene Anarchie anzusehen, als ein erbitterter Kampf ausschließlich ums Überleben, in dem sich jede Nation mit allen Mitteln und Kräften zu behaupten hätte. Diese radikale Deutung erklärt auch die von den chinesischen Intellektuellen wortmächtig propagierte Vorbildfunktion des deutschen Militarismus: Das scheinbar unaufhaltsame Vordringen der deutschen Truppen sollte als erfolgversprechendes Vorbild für ein China dienen, das von den Kolonialmächten zerstückelt, ja ausgelöscht $\mathrm{zu}$ werden drohte. Militarismus wurde als das geeignete Rezept für ein Land wahrgenommen, dessen Bürger oft als „kranke Männer Ostasiens“ verspottet wurden.

In seinem umfangreichen Aufsatz „Militarismus“ erklärt Liu Shuya mit klaren Worten und unter Bezugnahme auf Schopenhauers Begriff vom „Willen zum Leben“ die Notwendigkeit des Militarismus:

Warum ist heute so oft von Militarismus die Rede? Wegen des Willens zum Leben. Denn alles Leben stammt von diesem Willen und streitet um Raum, Zeit und Ressourcen, so dass dadurch der Lebenskampf entsteht. [. . .] Der Wille zum Leben ist der Ursprung der Welt, der Lebenskampf das Zentrum der Evolution. Der Staat besteht aus diesem Willen zum Leben. Militarismus ist die Spitze des Lebenskampfs. [. . .] Um das eigene Volk und Land zu schützen, kann der Staat von heute keinen anderen Weg nehmen als den Militarismus. Um nicht von anderen unterworfen zu werden, kann er kein anderes Mittel einsetzen als den Militarismus.

(Liu 1916b, 166)

Dem Militarismus wird insofern eine zwingende Notwendigkeit zugesprochen, damit das eigene Volk erhalten bleibt: Ist das reine Überleben das höchste Gebot in einer anarchistischen Weltlage, wo die Konkurrenz die Kooperation unmöglich macht, muss man mit militärischen Maßnahmen das eigene Existenzrecht verteidigen. Daher solle das deutsche Kaiserreich, das Liu zufolge „das stärkste Land auf der Welt“ sei, wieder „der Spiegel für uns“ werden (Liu 1916b, 167).

Die solchermaßen behauptete Vorbildfunktion des deutschen Militarismus wird nicht nur theoretisch, sondern auch historisch begründet. Vor allen Dingen wird der Aufstieg Preußens gerne als Beweis herangezogen. Im oben zitierten Aufsatz umreißt Liu Shuya ferner mit stilistischem Raffinement den Frieden von Tilsit, den Preußen und Russland im Juli 1807 unter großem Zugzwang mit Napoleon unterzeichnen mussten:

Wirft man einen Blick in die Geschichte, wird man sehen, dass Deutschland vor einem Jahrhundert noch viel schwächer und viel ärmer war als das heutige China. [. . .] Die mächtigen Truppen Napoleons zogen durch das Land und marschierten bis in den Norden. Dort im Norden fiel Königin Luise vor Napoleon auf die Knie und flehte ihn tränenvoll an - aber vergeblich. Der Sieger schenkte ihr kein Mitleid, so dass sie ihre bitteren Tränen herunterschlucken und Preußen in den Frieden von Tilsit einwilligen musste. Wegen dieses Friedens verlor Preußen die Hälfte von seiner Landfläche und Bevölkerung, 
musste eine Reparation in Höhe von 1.300 Millionen Franken zahlen, musste ferner die Größe seines stehenden Heers einschränken und durfte die Obergrenze von 42.000 Soldaten nicht überschreiten. Darüber hinaus hatte Preußen weiteren ungerechten Verträgen Folge zu leisten. Die erlittenen Demütigungen, Schmerzen und Verluste waren zehnfach so groß wie bei den Verträgen von Ja-Wu und Geng-Zi.

(Liu 1916b, 168)

Gemäß dem Vertrag von Ja-Wu im Jahre 1885, auch Friedensvertrag von Shimonoseki genannt, und dem Vertrag von Geng-Zi, darin im Jahre 1901 die Boxerentschädigung festgelegt wurde, musste China, ebenfalls wie das geschlagene Preußen, erniedrigende Bedingungen akzeptieren, unermessliche Zahlungen als Reparation leisten und den Kolonialmächten beträchtliche Teile seiner Souveränität wie etwa das Justiz- und Zollrecht preisgeben. Mögen die historischen Vergleiche in konkreten Statistiken einiges an Genauigkeit zu wünschen übriglassen, so lassen sie doch die Argumentationslogik der chinesischen Intellektuellen deutlich werden. Die historische Parallele zwischen China und Preußen, insbesondere der vergleichbare Ausgangspunkt, macht das Aufstiegspotential einer „verspäteten“ Nation durch den Weg des Militarismus plausibel. Bewusst wird die Erfolgsgeschichte Preußens gegen den erbärmlichen Zustand des unter den Warlords leidenden Chinas ausgespielt, um die Vorbildfunktion des deutschen Militarismus nochmals zu akzentuieren. Denn der „Hauptgrund für die Stärke Deutschlands“ liege Liu Shuya zufolge in einem umfassenden Militarismus, der auch kulturelle und geisteswissenschaftliche Bereiche einschließt. Als Träger dieser in jedwede Szene des intellektuellen Lebens eingedrungenen kriegerischen Mentalität werden u. a. Heinrich von Treitschke, Friedrich Paulsen, Rudolf Eucken und Gerhart Hauptmann genannt, wobei die beiden Letzteren jenes berüchtigte „Manifest der 93“ mitunterzeichnet haben, das mit dem Titel „An die Kulturwelt“ gewaltig erklärt: „Ohne den deutschen Militarismus wäre die deutsche Kultur längst vom Erdboden getilgt. [. . .] Deutsches Heer und deutsches Volk sind eins“ (vgl. Huang 2016).

\section{Kritik des deutschen Militarismus}

Allerdings hatte die Weltgeschichte bereits bewiesen, dass die unglückliche Kombination von Militarismus und Nietzsches Philosophie verheerende Konsequenzen hat. Der Bruch mit jeglichen normativen Traditionen und die Verabsolutierung des subjektiven Willensausdrucks kann zur Vernachlässigung der Rechte anderer führen. Auf die internationale Ebene übertragen, rechtfertigt es der auf Sozialdarwinismus basierende Militarismus, mit kriegerischen Mitteln rücksichtslos all das zu erobern, was zum eigenen Überdauern unabdingbar 
erscheint. Ab 1917 erkannten die chinesischen Intellektuellen allmählich, dass, wenn die Deutschen ihre aus dieser gefährlichen Mischung abgeleitete Weltpolitik konsequent durchgesetzt hätten, China als eine noch ziemlich schwache Nation sein Lebensrecht recht schnell verlieren würde - oder wie Liu Shuya selbst formuliert:

Unsere Jugend muss sich klarmachen: Die Deutschen haben unser Volk nur deshalb noch nicht aus der Welt getilgt, weil die Umstände es ihnen nicht erlaubten. Wenn aber eines Tags die Flugschiffe ostwärts hierher flögen, könnte eine einzige Division innerhalb von wenigen Monaten uns alle vierhundert Millionen Chinesen mit größter Leichtigkeit auslöschen.

(Liu 1916a, 98)

Neben der Einsicht in die verheerenden Konsequenzen eines uneingeschränkten Militarismus haben die chinesischen Intellektuellen um die Neue Jugend nach und nach auch die dahinterstehende rassistische Ideologie der Führungseliten des Deutschen Kaiserreichs erkannt. Selbst Liu Shuya, dieser ursprünglich so wortkräftige Propagandist des Militarismus, musste mit Erschrecken zur Kenntnis nehmen, dass die großen Köpfe der deutschen Wissenschaft ebenfalls Befürworter eines sozialdarwinistisch geprägten Rassismus waren. In seinem Aufsatz „Europäischer Krieg und das Bewusstsein der Jugend“ schreibt Liu:

Das größte Schrecknis für den Verfasser war jene Erklärung von R. Eucken und E. Haeckel, in der sie die Briten schonungslos verrissen haben. [. . .] Als der Europäische Krieg ausbrach, schrieben Eucken und Haeckel gemeinsam einen Aufruf und warfen den Briten öffentlich vor, trotz ihres teutonischen Stolzes die gelbe Rasse mit in den Krieg zu ziehen und mit einem halbasiatisch-halbbarbarischen Volk, nämlich den Russen, ein Bündnis zu schließen, um der eigenen Rasse Schaden zuzufügen. [. . .] Russland sei halbbarbarisch, weil es halbasiatisch sei - dies ist zwar eine feindliche Verleumdung, aber die Verachtung gegenüber unserem Ostasien und unserer gelben Rasse ist nicht zu verkennen. Ach! Wenn die großen Denker schon solch eine Ansicht vertreten, werden die Politiker und Kriegsmänner uns Ostasiaten noch als Menschen betrachten?

(Liu 1916a, 97)

Liu nimmt hier Bezug auf die „Erklärung“ von Eucken und Haeckel, die diese am 19. August 1914 in vielen deutschen Zeitungen abdrucken ließen. Zwar kommt in diesem Text die Formulierung „gelbe Rasse“ nicht vor, allerdings stellen die beiden Verfasser doch fest:

Aber was heute geschieht, ist doch das Stärkste von allem, es wird in den Annalen der Weltgeschichte dauernd als eine unauslöschliche Schande Englands bezeichnet werden. England kämpft zu Gunsten einer slawischen, halbasiatischen Macht gegen das Germanentum; es kämpft auf der Seite nicht nur der Barbarei, sondern auch des moralischen Unrechts [. . .].

(Eucken und Haeckel 1914) 
Hinter diesem Einwand gegen die Briten steht offenbar ein tiefverwurzelter Rassengedanke, dessen Echo sogar ins tausend Meilen entfernte China reichte. Anfang 1917 fasst Chen Duxiu in seinem Aufsatz „Die Außenbeziehung zu Deutschland“ zusammen: „Die weiße Rasse betrachtet unser Volk so, wie der Mensch Hunde und Pferde betrachtet. Insbesondere übertreiben die Deutschen ihren Patriotismus und nehmen überhaupt keine Rücksicht auf andere Völker“ (Chen 1917, 1). Da der mit Sozialdarwinismus und Rassenideologien vermengte Militarismus eine reale Bedrohung für China darstellt, soll sich die anzueignende Kriegsmentalität gegen den Lehrmeister richten. Daher spricht sich Chen entschieden für den Kriegseintritt gegen die Mittelmächte aus:

Der einzige Faktor für das Überleben eines Landes ist die militärische Kraft. [. . .] Wenn wir uns widerstandslos unterwürfen und die Schmach nur feige ertrügen, würden die kämpferischen und ehrbaren Deutschen, die die Macht am meisten schätzen, es nicht tolerieren, dass wir noch unter den Menschen fortexistierten, selbst wenn wir jeden Tag vor ihnen knien würden.

(Chen 1917, 3)

Neben dem rassistisch geprägten Militarismus wurde auch verstärkt Kritik an der Philosophie Nietzsches geübt. Die Außerkraftsetzung regulierender gesellschaftlicher Normen ermöglicht zwar die freie Entgrenzung der Subjektivität und realisiert die Emanzipation des Individuums. Jedoch kann die amoralische Handlungsmaxime auch dazu führen, das Recht anderer $\mathrm{zu}$ ignorieren und sogar einen unbedingten Machtanspruch gegenüber allen eigentlich Gleichberechtigten zu erheben. Eine einseitige, verabsolutierende Deutung der Philosophie Nietzsches, insbesondere jener verführerischen Begriffe des „Willens zur Macht“ oder des „Übermenschen“, bahnt den Weg zur imperialistischen Machtbesessenheit und Hegemonie. Diese Gefahr der verhängnisvollen Verschmelzung von Militarismus, Sozialdarwinismus und einer auf den „Willen zur Macht“ fixierten ideologischen Auslegung der Philosophie Nietzsches haben die Autoren der Neuen Jugend auch im Laufe der Zeit deutlich erkannt. So schreibt Hu Shi in seinem in dieser Zeitschrift abgedruckten Tagebuch:

Die größte Gefahr von heute ist ein Nationalismus im engsten Sinne, der meint, dass das eigene Land über allen anderen stehen soll, und das eigene Volk ebenfalls. (So heißt es in der deutschen Nationalhymne: Deutschland, Deutschland, über alles.) Man scheut nicht, alle Mittel, die zu diesem egoistischen Zweck führen könnten, einzusetzen, selbst wenn dabei ein anderes Land vernichtet oder ein anderes Volk zerstückelt werden würde. [. . .] Man bildet sich ein, die Gewalt wäre das geltende Gesetz zwischen den Nationen, und das internationale Recht bedeutete nichts anderes, als dass die Schwächeren zum Futter für die Stärkeren dienten.

(Hu 1917b, 345)

Zitiert wird hier die Eingangszeile des „Lieds der Deutschen“ von Hoffmann von Fallersleben, die heute offiziell nicht mehr gesungen wird. Hu Shi stellt in 
dieser Passage die Logik des Machtgedankens bloß, die um der eigenen Priorität und Vorreiterstellung willen die ethischen und juristischen Regulierungen außer Acht lässt und das Recht der Anderen untergräbt. Hu Shi führt ferner die Wurzel dieses radikalen Anspruchs auf die Philosophie Nietzsches zurück, die seiner Meinung nach jegliche moralische Kultur unter dem Vorwand des „Überlebens“ dezidiert ablehnt:

\begin{abstract}
Derjenige, der am lautstärksten die Philosophie der Macht vertrat, war der Deutsche Nietzsche. [. . .] So sagt er, dass der Sinn des Lebens nicht im Leben an sich liege, sondern im Willen zur Macht. Das Ziel der Menschheit bestehe demgemäß auch darin, eine Gesellschaft von „Übermenschen“ zu konstruieren. Die „Übermenschen“ seien die starken Menschen. Alle schwachen gehörten zu den Auszurottenden. Man solle diese vertilgen, vernichten, so dass keine Nachkommen überlebten. Die Welt gehöre den Stärkeren. Die sogenannte Moral sowie Gesetz, Mitleid und Frieden dienten nur dem Schutz der Schwächeren vor der zermalmenden Macht der Stärkeren, seien also die großen Feinde der Menschheit. [. . . ] Seit Nietzsches Lehren gibt es keine Ethik mehr auf der Welt.
\end{abstract}

(Hu 1917b, 345-346)

Auch Cai Yuanpei, der ehemalige Dekan der Peking Universität (Abb. 5), welche die Wiege der 4.-Mai-Bewegung war, hat an der fragwürdigen Kombination von Militarismus und Nietzsches Philosophie Anstoß genommen. In seinem Aufsatz „Der Europäische Krieg und die Philosophie“ schreibt Cai:

Die Weltanschauung Nietzsches ist also ein reiner Wille, der sich ausschließlich an der Macht orientiert. Deshalb sagt er: „Es gibt kein Gesetz. Es gibt keine Ordnung.“ Seine Lehren sind aristokratisch anstatt demokratisch, daher wurden sie von der aristokratischen Regierung Deutschlands ausgenutzt und in den Dienst des Militarismus gestellt. Auch wurde überall laut ,Deutsche über alles‘ gesungen - das ist doch die Lehre vom „Übermenschen“. [. . .] Verträge waren wertloses Papier - das ist doch eine Lehre jenseits des Rechts. Überblickt man die Politik des deutschen Kaiserreichs während der Kriegszeit, würde man nichts finden, was nicht mit Nietzsches Philosophie übereinkäme.

(Cai 1918, 379)

Sowohl Hu Shi als auch Cai Yuanpei haben erkannt, dass die Theorie Nietzsches vom Militarismus sozialdarwinistisch gedeutet wurde, um die eigene Hegemonie und den kriegerischen Weg dahin zu legitimieren. Der Individualismus verzerrt sich zum Egoismus der Nation, die Kritik an veralteter Kulturtradition zur Nivellierung der regulierenden Instanzen, die Entfesslung der Subjektivität zu unkontrollierten Gewalttaten. Deshalb stellt Tao Lügong, der etliche Beiträge an die Neue Jugend geliefert hat, Anfang 1919 fest: „Der Militarismus ist eine absurde Idee, weil sie schon den Keim ihrer Niederlage in sich enthält, weil sie das Leben und den Wert des Lebens verneint“ (Tao 1919, 40-41). Insofern lässt sich beobachten, dass sich die ursprüngliche Begeisterung für den deutschen Militarismus und für die Philosophie Nietzsches schon hier in Kritik gewandelt hat. 


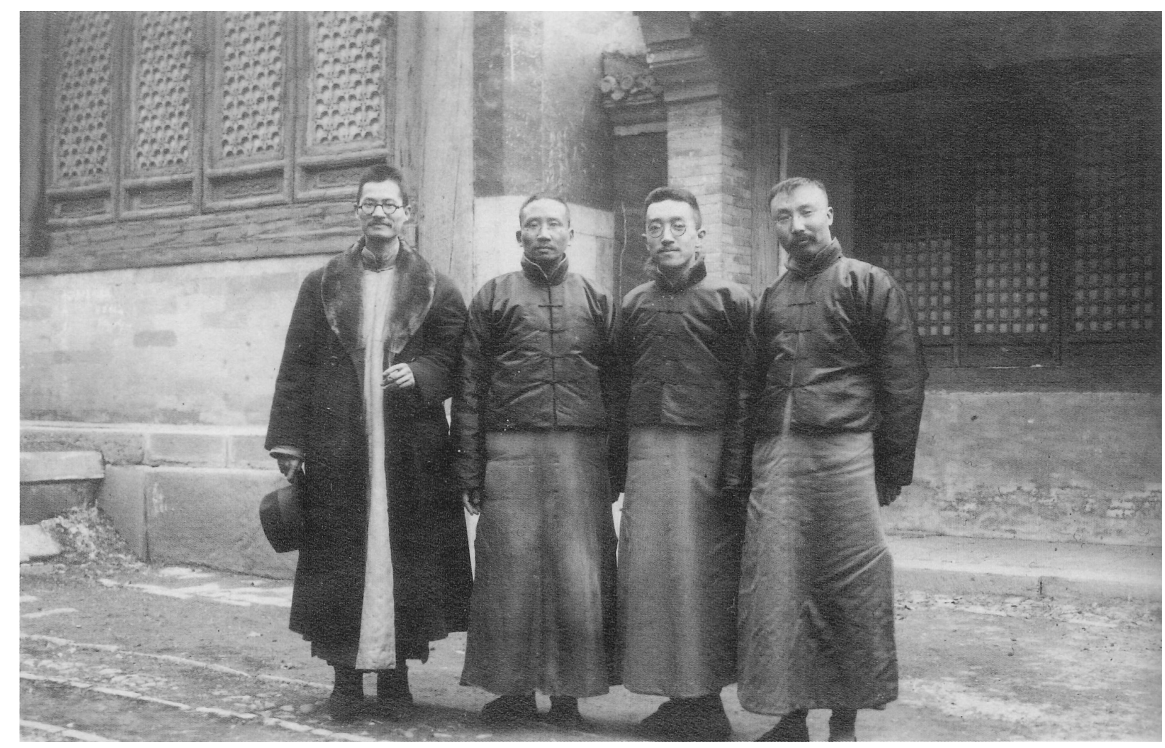

Abb. 5: Gruppenfoto von Jiang Menglin (1886-1964), Cai Yuanpei (1868-1940), Hu Shi (1891-1962) und Li Dazhao (1889-1927).

\section{Soziale Revolution und Marxismus}

Aber die scharfe Kritik am Militarismus und Nietzsches Philosophie bedeutet noch nicht, dass Deutschland und seine Vorbildfunktion für China insgesamt in Frage gestellt würden. Neben dem von Militarismus und rassistischen Ressentiments geprägten Kaiserreich tritt ein weiteres Deutschland hervor, das wegweisend für die zukünftige Entwicklung Chinas sein soll. Das ist das Deutschland des Sozialismus und Marxismus. Für die chinesischen Intellektuellen war das Ende des Ersten Weltkriegs nicht der Sieg einer militärischen Koalition über eine andere, sondern die Ablösung des deutschen Militarismus durch den deutschen Sozialismus. Chen Duxiu schreibt im Oktober 1918 in seinem Aufsatz „Der VonKetteler-Bogen“: „Wir feiern jetzt nicht den militärischen Sieg der Entente über die Mittelmächte, sondern den politischen Fortschritt Deutschlands“ (Chen 1918b, 370). Und in seiner heute viel gelesenen Rede „Der Sieg des Bolschewismus“, die Li Dazhao unmittelbar nach dem Kriegsende auf dem Platz des Himmlischen Friedens hielt, vertritt er dieselbe Ansicht:

Der wahre Grund für die Beendigung des Kriegs besteht nicht etwa darin, dass die Entente die Mittelmächte militärisch besiegt hat, sondern darin, dass der deutsche Sozialis- 
mus den deutschen Militarismus überwunden hat. [. . .] Diese große Errungenschaft [. . . ] ist das Werk von Liebknecht, von Scheidemann, vor allem aber das Werk von Marx. Wenn wir diese entschiedene Weltveränderung feiern, sollen wir vor allem den Triumph der Demokratie über die Monarchie, den Triumph des Sozialismus über den Militarismus feiern.

(Li 1918, 365-366)

In dieser berühmten Rede entwirft Li Dazhao, dieser erste große Verfechter des kommunistischen Ideals in China, ein zweifaches Deutschlandbild: auf der einen Seite jener früher vielgepriesene, jetzt aber am Boden liegende Militarismus, auf der anderen Seite Demokratie und Sozialismus. Letztere bedeuten für Li Dazhao zweifelsohne die Hoffnung auf eine neue Welt. Das Interesse für die sozialistische Bewegung in Deutschland war schon immer präsent in den ersten Jahrgängen der Neuen Jugend. Beispielsweise hat Li Yimin schon im zweiten Heft des ersten Jahrgangs den Aufstieg der SPD geschildert, der sich im ersten Jahrzehnt des 20. Jahrhunderts trotz der Unterdrückung durch die preußischen Behörden vollzog (Li 1915b, 151).

Die Neue Jugend war aber auch das Organ der Neue-Kultur-Bewegung und der literarischen Revolution und hat in dieser Funktion Meisterwerke der Weltliteratur teils übersetzt, teils durch Kommentare und Inhaltsangaben bei den chinesischen Lesern bekannt gemacht. $\mathrm{Zu}$ den in der Zeitschrift präsentierten ausländischen Literaturströmungen gehört auch der deutsche Naturalismus. In seinen Tagebuchauszügen stellt Hu Shi unter anderem Die Weber von Gerhart Hauptmann als ein Werk vor, das eine revolutionäre Situation dramatisch vergegenwärtigt. Hu Shi beschreibt die einzelnen Szenen aus diesem Stück, fasst die Thematik unter der Überschrift „Die Ungleichheit zwischen Arm und Reich“ zusammen und bemerkt, dass die Darstellung der Armut unter den Webern „einen wirklich zum Weinen bringt“. Am Ende seiner Einführung stellt Hu Shi fest:

Es gehört zu der Erkenntnis einer neuen Zeit, dass die Kluft zwischen Armut und Reichtum von menschlichem Tun herrührt, und dass diese darum von Menschen abgeschafft werden kann. Jetzt wissen die Arbeiter, dass sie mit geeinter Kraft die Kapitalisten in die Enge zwingen. Deshalb haben sie sich verbündet und setzen alles aufs Spiel, nur um sich zu retten.

(Hu 1917a, 357)

Durch landeskundliche und literarische Vermittlung haben also wesentliche Momente des Sozialismus in China Fuß gefasst. Nach dem Ende des Ersten Weltkriegs und unmittelbar vor der 4.-Mai-Bewegung war das Deutschlandbild in der Neuen Jugend deshalb immer noch positiv besetzt, weil es nun von Sozialismus und Marxismus geprägt war. Für die chinesischen Intellektuellen war Deutschland immer noch das große Vorbild, aber es ist ein anderes Deutsch- 
land als das des Militarismus. Exemplarisch hierfür ist das fünfte Heft des sechsten Jahrgangs, das im Mai 1919 erschien. In dieser von Li Dazhao herausgegebenen Ausgabe, die im Nachhinein „Sonderheft Marxismus“ genannt wurde (Abb. 6), sind viele einführende Texte über Marx und seine Theorie veröffentlicht, darunter der berühmte Aufsatz des Herausgebers mit dem Titel „Meine Ansicht über den Marxismus“ (Li 1919). In diesem Text stellt Li Dazhao den Geschichtsmaterialismus und die Lehre vom Klassenkampf vor und übersetzt Auszüge des Kommunistischen Manifests aus der japanischen Übertragung von Hajime Kawakami (1879-1946) ins Chinesische. Li Dazhao wirbt für einen Arbeiterzusammenschluss sowie für die soziale Revolution und betrachtet das Volk als den eigentlichen Protagonisten des gesellschaftlichen Fortschritts:

Gemeinsam verfassten Marx und Engels das Kommunistische Manifest, in dem sie lautstark die Arbeiterklasse aller Welt aufrufen, sich zu vereinen und den Kapitalismus zu untergraben. Erst dann weiß man, dass der Sozialismus ohne das Volk nie realisiert werden kann. Das ist ein großes Verdienst des Marxismus.

(Li 1919, 468)

Seit dem Erscheinen dieses Sonderheftes verlagerte sich der Fokus der Neuen Jugend nach und nach auf jenes Land, in dem der Marxismus seither praktiziert wurde. Vom achten Jahrgang 1920 an wurde die Neue Jugend zur offiziellen Publikation der kurz zuvor in Shanghai gegründeten „Marxistischen Gruppe“, aus der dann die Kommunistische Partei Chinas hervorging. Es wurden zahlreiche Beiträge aus der amerikanischen Zeitschrift Soviet Russia übersetzt. Li Dazhao selbst rief an der Peking Universität eine „Gesellschaft zum Studium des Marxismus“ ins Leben und widmete sich der Verbreitung des Marxismus in China. Und das Deutschland der Weimarer Republik, das gerade den Spartakusaufstand unterdrückt und den Kapp-Putsch abgewehrt hatte, verschwand allmählich aus dem Horizont der Neuen Jugend. 


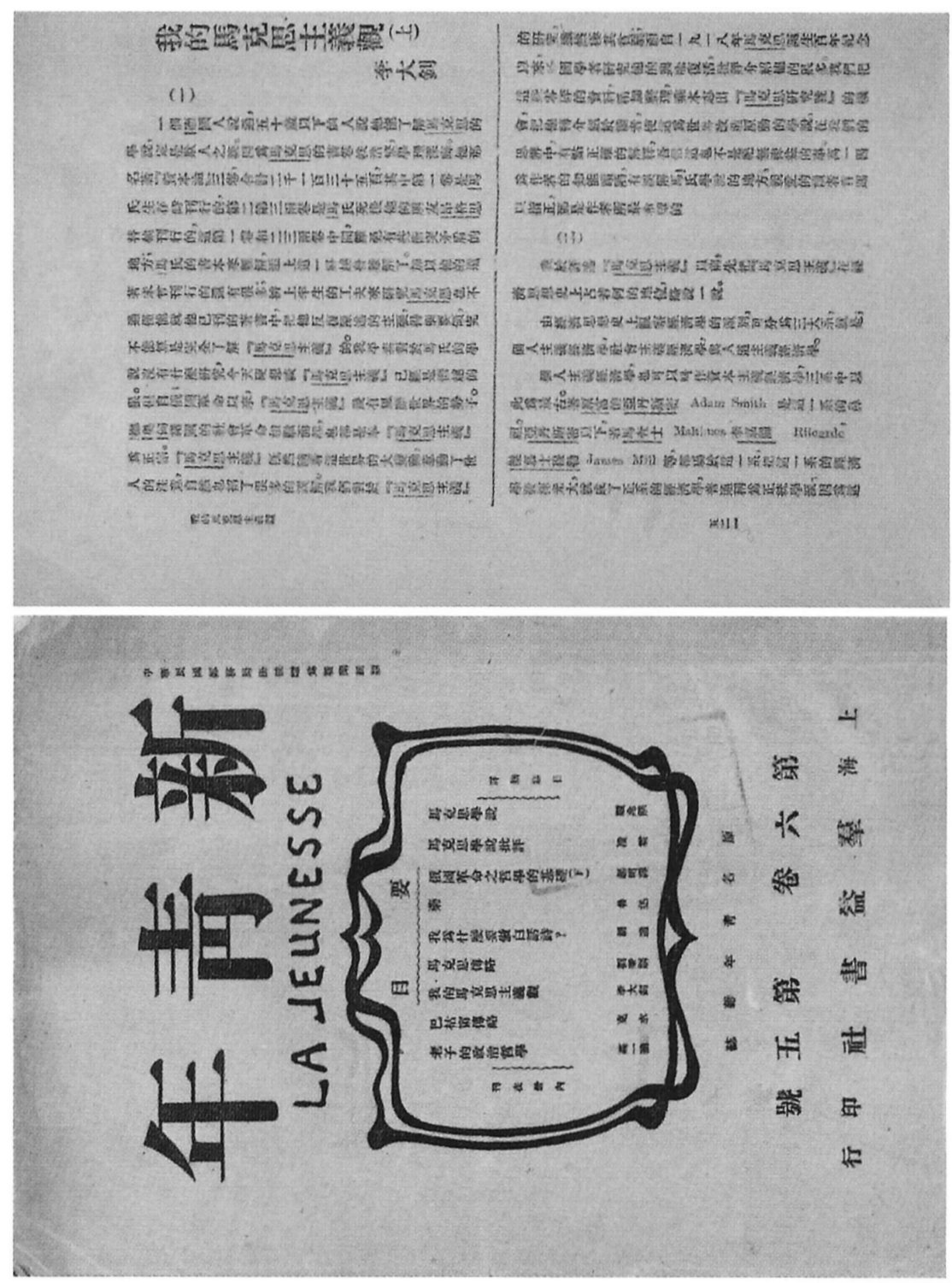

Abb. 6: Das Marxismus-Sonderheft der Neuen Jugend. 


\section{Literaturverzeichnis}

Cai, Yuanpei. 蔡元培. Ou zhan yu zhe xue. 欧战与哲学. „Europäischer Krieg und die Philosophie“. Zeitschrift für die Jugend 5.5 (1918). Reprint der „Neuen Jugend“ in 12 Bänden. Fünfter Band. Beijing 2011. 378-381.

Cao, Weidong. 曹卫东. De guo qing nian yun dong. 德国青年运动. Die deutsche Jugendbewegung. Shanghai 2013.

Chen, Duxiu. 陈独秀. Ren sheng zhen yi. 人生真义. „Über den wahren Sinn des Lebens“. Zeitschrift für die Jugend 4.2 (1918a). Reprint der „Neuen Jugend“ in 12 Bänden. Vierter Band. Beijing 2011. 71-73.

Chen, Duxiu. 陈独秀. Ke lin de bei. 克林德碑. „Der Von-Ketteler-Bogen“. Zeitschrift für die Jugend 5.5 (1918b). Reprint der „Neuen Jugend“ in 12 Bänden. Fünfter Band. Beijing 2011. 370-377.

Chen, Duxiu. 陈独秀. Dui de wai jiao. 对德外交. „Die Außenbeziehung zu Deutschland“. Zeitschrift für die Jugend 3.1 (1917). Reprint der „Neuen Jugend“ in 12 Bänden. Dritter Band. Beijing 2011. 1-3.

Chen, Duxiu. 陈独秀. Yi jiu yi liu nian. 一九一六年. „Das Jahr 1916“. Zeitschrift für die Jugend 1.5 (1916). Reprint der „Neuen Jugend“ in 12 Bänden. Erster Band. Beijing 2011. 305-307.

Chen, Duxiu. 陈独秀. Jing gao qing nian. 敬告青年. „Aufruf an die Jugend“. Zeitschrift für die Jugend 1.1 (1915a). Reprint der „Neuen Jugend“ in 12 Bänden. Erster Band. Beijing 2011.1-5.

Chen, Duxiu. 陈独秀. Fa lan xi ren yu jin shi wen ming. 法兰西人与近世文明. „Die Franzosen und die moderne Zivilisation“. Zeitschrift für die Jugend 1.1 (1915b). Reprint der „Neuen Jugend“ in 12 Bänden. Erster Band. Beijing 2011. 6-8.

Chen, Duxiu. 陈独秀. Dong xi min zu gen ben si xiang zhi cha yi. 东西民族根本思想之差异. „Die grundlegenden Mentalitätsunterschiede zwischen östlichen und westlichen Nationen“. Zeitschrift für die Jugend 1.4 (1915c). Reprint der „Neuen Jugend“ in 12 Bänden. Erster Band. Beijing 2011. 231-233.

Chow, Tse-tsung. May Fourth Movement. Intellectual Revolution in Modern China. Cambridge, MA 1960.

Eucken, Rudolf, und Ernst Haeckel. „Erklärung“. Jenaer Volksblatt, 20. August 1914. https:// zs.thulb.uni-jena.de/rsc/viewer/jportal_derivate_00246143/JVB_19140820_194_ 167758667_B1_02.tif [6. April 2020].

Felber, Roland. „Das chinesische Deutschlandbild in der Zeit des Vierten Mai“. Berliner ChinaHefte 17 (1999): 27-40.

Gálik, Marián. From Goethe, Nietzsche to Rilke. Studies in Sino-German interliterary process. Hrsg. Yan Liu. Fuzhou 2017.

$\mathrm{Hu}$, Shi. 胡适. Xin si chao de yi yi. 新思潮的意义. „Die Bedeutung der neuen Denkströmung“. Zeitschrift für die Jugend 7.1 (1919). Reprint der „Neuen Jugend“ in 12 Bänden. Siebter Band. Beijing 2011. 4-9.

Hu, Shi. 胡适. Cang hui shi zha ji. Xu qian hao. 藏轫室札记. 续前号. „Notizen aus der Nische des verborgenen Lichts. Fortsetzung“. Zeitschrift für die Jugend 2.5 (1917a). Reprint der „Neuen Jugend“ in 12 Bänden. Zweiter Band. Beijing 2011. 356-359.

$\mathrm{Hu}$, Shi. 胡适. Cang hui shi zha ji. Xu qian hao. 藏轫室札记. 续前号. „Notizen aus der Nische des verborgenen Lichts. Fortsetzung“. Zeitschrift für die Jugend 3.5 (1917b). Reprint der „Neuen Jugend“ in 12 Bänden. Dritter Band. Beijing 2011. 345-349.

Huang, Liaoyu. 黄燎宇. Dang na xiong nai er de ge sheng xiang che de yi zhi da di. „Gao wen ming shi jie shu“ shu ping. 当纳雄耐尔的歌声响彻德意志大地 - ”告世界文明书“述评. 
„Wenn das Lied des Nationalen über Deutschland tönt. Kommentar zum Aufruf an die Kulturwelt." Deutschlandstudien der Peking Universität 5 (2016): 15-25.

Kirby, William. Germany and Republican China. Stanford, CA 1984.

Leutner, Mechthild, und Andreas Steen. Deutsch-chinesische Beziehungen 1911-1927. Vom Kolonialismus zur Gleichberechtigung. Eine Quellensammlung. Berlin 2006.

Li, Dazhao. 李大钊. Wo de ma ke si zhu yi guan. 我的马克思主义观. „Meine Ansicht über den Marxismus“. Zeitschrift für die Jugend 6.5 (1919). Reprint der „Neuen Jugend“ in 12 Bänden. Sechster Band. Beijing 2011. 455-471.

Li, Dazhao. 李大钊. Bolshevism de sheng li. Bolshevism的胜利. „Der Sieg des Bolschewismus“. Zeitschrift für die Jugend 5.5 (1918). Reprint der „Neuen Jugend“ in 12 Bänden. Fünfter Band. Beijing 2011. 365-369.

Li, Yimin. 李亦民. Shi jie shuo yuan. 世界说苑. „Aus aller Welt“. Zeitschrift für die Jugend 1.1 (1915a). Reprint der „Neuen Jugend“ in 12 Bänden. Erster Band. Beijing 2011. 69-75.

Li, Yimin 李亦民. Shi jie shuo yuan. 世界说苑. „Aus aller Welt“. Zeitschrift für die Jugend 1.2 (1915b). Reprint der „Neuen Jugend“ in 12 Bänden. Erster Band. Beijing 2011. 150-152.

Liang, Qichao. 梁启超. Xin min shuo. 新民说. Der neue Bürger. Hrsg. Zhiming Song. Shenyang 1994.

Liu, Shuya. 刘叔雅. Ou zhou zhan zheng yu qing nian zhi jue wu. 欧洲战争与青年之觉悟. „Europäischer Krieg und das Bewusstsein der Jugend“. Zeitschrift für die Jugend 2.2 (1916a). Reprint der „Neuen Jugend“ in 12 Bänden. Zweiter Band. Beijing 2011. 93-99.

Liu, Shuya. 刘叔雅. Jun guo zhu yi. 军国主义. „Militarismus“. Zeitschrift für die Jugend 2.3 (1916b). Reprint der „Neuen Jugend“ in 12 Bänden. Zweiter Band. Beijing 2011. 166-171.

Lu, Xun. 鲁迅. Ren zhi li shi. De guo hei ge er shi zhong zu fa sheng xue zhi yi yuan yan jiu quan jie. 人之历史. 德国黑格尔式种族发生学之一元研究诠解. „Die Geschichte der Menschheit. Deutung der Phylogene-Lehre von Haeckel aus Deutschland“. Sämtliche Werke. Band 1. Beijing 2005. 8-17.

Meinecke, Friedrich. „Die deutsche Katastrophe“. Autobiographische Schriften. Friedrich Meinecke. Hrsg. Eberhard Kessel. Stuttgart 1969. 323-445.

Pan, Zan. 潘赞. De guo jun shen xing deng bao yuan shuai. 德国军神兴登堡元帅. „Der deutsche Kriegsgott Feldmarschall von Hindenburg“. Zeitschrift für die Jugend 1.4 (1915). Reprint der „Neuen Jugend“ in 12 Bänden. Erster Band. Beijing 2011. 282-284.

Pusey, James Reeve. China and Charles Darwin. Cambridge, MA 1983.

Tao, Lügong. 陶履恭. Jun guo zhu yi. 军国主义. „Militarismus“. Monatsschrift der Peking Universität. 1.1 (1919): 33-44.

Wang, Zhongjiang. 王中江. Jin hua zhu yi zai zhong guo. 进化主义在中国. Der Evolutionismus in China. Beijing 2010.

Xie, Hong. 谢鸿. De guo qing nian tuan. 德国青年团. „Der Jungdeutschland-Bund“. Zeitschrift für die Jugend 1.3 (1915). Reprint der „Neuen Jugend“ in 12 Bänden. Erster Band. Beijing 2011. 193-195.

Yue, Daiyun. 乐黛云. Ni cai yu zhong guo xian dai wenxue. 尼采与中国现代文学. „Nietzsche und die moderne chinesische Literatur“. Wissenschaftliche Zeitung der Peking Universität 17.3 (1980): 20-33.

Zhao, Bing. 赵兵. Ou zhan qian hou zhong guo xin wen hua yun dong zhong de „de yi zhi jing shen“ji qi zhuan xiang. 欧战前后中国新文化运动中的“德意志精神”及其转向. „Der, deutsche Geist" und seine Wendung in der Neuen-Kultur-Bewegung vor und nach dem Europäischen Krieg“. Deutschlandstudien 32.1 (2017): 102-117. 


\section{Bildquellen}

Abbildungen 1, 2, 5, 6

Abbildungen 3, 4
Lu Xun Museum (Beijing New Culture Movement Memorial)

Rara-Zeitschriften-Abteilung der Bibliothek der Peking Universität 
